\title{
MR Imaging Criteria for the Detection of Nasopharyngeal Carcinoma: Discrimination of Early-Stage Primary Tumors from Benign Hyperplasia
}

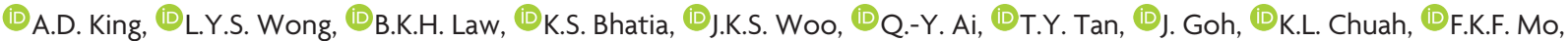 \\ (D).C.A. Chan, D.T.C. Chan, and (D)A.C. Vlantis
}

\begin{abstract}
BACKGROUND AND PURPOSE: MR imaging can detect nasopharyngeal carcinoma that is hidden from endoscopic view, but for accurate detection carcinoma confined within the nasopharynx (stage T1) must be distinguished from benign hyperplasia of the nasopharynx. This study aimed to document the MR imaging features of stage T1 nasopharyngeal carcinoma and to attempt to identify features distinguishing it from benign hyperplasia.
\end{abstract}

MATERIALS AND METHODS: MR images of 189 patients with nasopharyngeal carcinoma confined to the nasopharynx and those of 144 patients with benign hyperplasia were reviewed and compared in this retrospective study. The center, volume, size asymmetry (maximum percentage difference in area between the right and left nasopharyngeal halves), signal intensity asymmetry, deep mucosal white line (greater contrast enhancement along the deep tumor margin), and absence/distortion of the adenoidal septa were evaluated. Differences were assessed with logistic regression and the $\chi^{2}$ test.

RESULTS: The nasopharyngeal carcinoma center was lateral, central, or diffuse in 134/189 (70.9\%), 25/189 (13.2\%), and 30/189 (15.9\%) cases, respectively. Nasopharyngeal carcinomas involving the walls showed that a deep mucosal white line was present in 180/183 (98.4\%), with a focal loss of this line in 153/180 (85\%) cases. Adenoidal septa were absent or distorted in $111 / 111$ (100\%) nasopharyngeal carcinomas involving the adenoid. Compared with benign hyperplasia, nasopharyngeal carcinoma had a significantly greater volume, size asymmetry, signal asymmetry, focal loss of the deep mucosal white line, and absence/distortion of the adenoidal septa $(P<.001)$. Although size asymmetry was the most accurate criterion (89.5\%) for nasopharyngeal carcinoma detection, use of this parameter alone would have missed $11.9 \%$ of early-stage $\mathrm{Tl}$ nasopharyngeal carcinomas.

CONCLUSIONS: MR imaging features can help distinguish stage T1 nasopharyngeal carcinoma from benign hyperplasia in most cases.

ABBREVIATIONS: $\mathrm{BH}=$ benign hyperplasia; $\mathrm{BH} 1=\mathrm{BH}$ type $1 ; \mathrm{BH} 2=\mathrm{BH}$ type $2 ; \% \Delta \mathrm{A}=$ maximum percentage difference in area; $\mathrm{NPC}=$ nasopharyngeal carcinoma

U ndifferentiated nasopharyngeal carcinoma (NPC) is highly responsive to radiation therapy and often curable when detected at an early stage. Endoscopy remains the standard investigation of primary tumor detection, followed by endoscopic biopsy for histologic confirmation. However, endoscopic visualization is not possible for all tumors, including small nasopharyngeal tumors

Received April 26, 2017; accepted after revision October 28.

From the Departments of Imaging and Interventional Radiology (A.D.K., L.Y.S.W. B.K.H.L., Q.-Y.A.), Clinical Oncology (F.K.F.M., A.T.C.C.), and Otorhinolaryngology, Head and Neck Surgery (J.K.S.W., A.C.V.); and Department of Chemical Pathology (K.C.A.C.), State Key Laboratory in Oncology in South China, Li Ka Shing Institute of Health Sciences, Faculty of Medicine, The Chinese University of Hong Kong, Prince of Wales Hospital, Hong Kong, S.A.R., China; Imaging Department (K.S.B.), St Mary's Hospital, Imperial College Healthcare, National Health Service Trust, London, UK; Department of Radiology (T.Y.T.), Changi General Hospital, Singapore; and Departments of Diagnostic Radiology (J.G.) and Pathology (K.L.C.), Tan Tock Seng Hospital, Singapore.

This work was supported, in part, by grants from the Research Grants Council of the Hong Kong Special Administrative Region, China (project No. CUHK4457/06, CUHK4656/12, and SEG_CUHK02). in the lateral nasopharyngeal recess or a submucosal location. Our previous research demonstrated the importance of MR imaging as an adjunct to endoscopy during NPC detection. Overall, MR imaging-based detection of NPC is very accurate (95\%); additionally, $10 \%$ of MR imaging-detected NPCs are endoscopically occult, and MR imaging can detect slow-growing tumors up to 3 years before endoscopic detection. ${ }^{1-3}$ However, earlier MR imaging research was conducted in a largely symptomatic group of patients with NPC, which included those with advanced local disease in whom NPC detection had been assisted by submucosal tumor spread into the deep tissues surrounding the nasopharynx.

Please address correspondence to A.D. King, FRCR, Department of Imaging and Interventional Radiology, Faculty of Medicine, The Chinese University of Hong Kong, Prince of Wales Hospital, 30-32 Ngan Shing St, Shatin, New Territories, Hong Kong S.A.R. China; e-mail: king2015@cuhk.edu.hk

- Indicates open access to non-subscribers at www.ajnr.org

E Indicates article with supplemental on-line table.

http://dx.doi.org/10.3174/ajnr.A5493 
By contrast, the detection of early-stage T1 tumors confined within the nasopharynx, which must also be discriminated from benign hyperplasia $(\mathrm{BH})$ along the nasopharyngeal walls and in the adenoid, presents a greater challenge to MR imaging. Currently, the use of MR imaging as an adjunct for the detection of stage T1 NPC is increasing as more asymptomatic patients with early-stage cancers are detected through screening programs that use plasma markers such as Epstein-Barr virus DNA. ${ }^{4-7}$

Although size asymmetry between the right and left nasopharyngeal halves and loss of the normal adenoidal septa are among the features used to detect NPC via MR imaging, ${ }^{1,2,8}$ the incidence of each sign has not been assessed. In addition, with a greater focus on early NPC detection through screening programs, we have become more aware of the range of MR imaging features that may be seen in NPC confined to the nasopharynx. These MR imaging features, which include the presence and integrity of a deep mucosal line along the walls of the nasopharynx (ie, a line of greater enhancement along the deep layer of the tumor) and variations in signal intensity and heterogeneity, have received scant attention in the literature. We have also observed that some of these MR imaging features of stage T1 NPC overlap those of $\mathrm{BH}$; thus potentially reducing the accuracy of MR imaging for NPC detection.

In this era of screening programs for early NPC detection, our first aim was to document the MR imaging features of NPC confined to the nasopharynx or early-stage T1 lesions, which are the least obvious of the $4 \mathrm{~T}$-stages on MR images. Our second aim was to compare these MR imaging features with those of $\mathrm{BH}$ to determine whether our current MR imaging criteria ${ }^{2}$ require refinement to improve NPC detection.

\section{MATERIALS AND METHODS \\ Patients}

This retrospective study was approved by the Chinese University of Hong Kong institutional review board, and the requirement for written informed consent from the patients was waived. The NPC and non-NPC groups included 28 and 160 patients, respectively, who participated in a previous study ${ }^{2}$ and long-term follow-up analysis $^{3}$ to compare the diagnostic accuracy of MR imaging with that of endoscopy and endoscopic biopsy.

\section{NPC Group}

The nasopharyngeal MR images of 212 consecutive patients with primary NPC tumors confined to nasopharynx (stage T1) who underwent contrast-enhanced MR imaging between January 2005 and June 2014 were reviewed. The post-nasopharyngeal biopsy MR images of 23 patients were degraded, leaving 189 patients for analysis (141 men, 48 women; mean age, 52.5 years; age range, 23-80 years). Among these cases, 188 were undifferentiated carcinoma and one was a poorly differentiated carcinoma.

\section{BH Group}

The nasopharyngeal MR images of 206 consecutive patients who were investigated for suspected NPC but had no endoscopic evidence of NPC at the time of contrast-enhanced MR imaging, between July 2005 and June 2014, and after a minimum follow-up of 2 years were reviewed. Of these, $62(30.1 \%)$ patients with a normal nasopharynx, defined as a mucosal thickness of $\leq 3 \mathrm{~mm}$ plus an adenoid that was absent, or vestigial (contrast-enhancing septa with little or no intervening solid tissue) or comprising cysts (including a Tornwaldt cyst) were excluded from the study, leaving 144 patients with benign hyperplasia for analysis ( 84 men, 60 women; mean age, 48.2 years; age range, $17-83$ years). $\mathrm{BH}$ presents in 2 main patterns with different MR imaging features and was therefore divided into types $1(\mathrm{BH} 1)$ and 2 (BH2) based on the dominant pattern on T1-weighted postcontrast images, according to previously described criteria. ${ }^{2}$ Essentially, BH1 comprised a mucosal thickness of $>3 \mathrm{~mm}$ with homogeneous contrast enhancement and/or an adenoid without contrast-enhancing septa and was present in 66/144 cases (45.8\%). BH2 comprised a mucosal thickness of $>3 \mathrm{~mm}$ with a line/band of greater contrast enhancement in the deep layer relative to the superficial layer of the wall (deep mucosal white line) and/or an adenoidal mass with contrast-enhancing septa separated by linear bands of minimally contrast-enhancing tissue (striped appearance) and was present in $78 / 144$ cases $(54.2 \%)$.

\section{MR Imaging Acquisition}

MR imaging of the nasopharynx was performed on a $1.5 \mathrm{~T}$ or $3 \mathrm{~T}$ whole-body MR imaging system. The examination targeted the nasopharynx, and approximately 20 minutes were required to perform the following 4 sequences in the MR imaging protocol: an axial fat-suppressed T2-weighted sequence (TR/TE, 2500/100 ms; echo-train length, 15; FOV, 22 cm; section thickness, $4 \mathrm{~mm}$ with no intersection gap; matrix, $256 \times 202)$, an axial T1-weighted spin-echo sequence (TR/TE, 500/20 ms; FOV , $22 \mathrm{~cm}$; section thickness, $4 \mathrm{~mm}$ with no intersection gap; matrix, $512 \times 512$ ), and a T1-weighted spin-echo sequence in the axial and coronal planes following a bolus injection of 0.1 mmol of gadoterate meglumine (Dotarem; Guerbet, Aulnaysous-Bois, France) per kilogram of body weight.

\section{MR Imaging Analysis}

The nasopharynx was divided into 2 main sites: the nasopharyngeal walls and the adenoid, which consisted of a focal bulge in the roof/posterior wall. NPC was assessed regarding the following parameters: site (walls, adenoid, subsites, and tumor center), size (volume and size asymmetry), signal intensity (intensity, homogeneity, and asymmetry), deep mucosal white line, and adenoidal septa as defined in Table 1. Cysts were excluded from the analysis of MR imaging features related to signal intensity and asymmetry. $\mathrm{BH}$ was assessed regarding the following parameters: site (walls and adenoid), size (volume and size asymmetry), signal intensity (intensity and signal asymmetry), deep mucosal white line (walls), and adenoidal septa (adenoid), as described for NPC. For the investigation of signal intensity, $\mathrm{BH}$ was divided into $\mathrm{BH} 1$ and $\mathrm{BH} 2$, and the representative $\mathrm{ROI}$ on each $\mathrm{T} 1$-weighted postcontrast image was obtained from either the enhancing wall of a $\mathrm{BH} 1$ or from the band of low contrast enhancement in the wall or adenoidal columns of a BH2. By definition, the deep mucosal white line is not present in $\mathrm{BH} 1$ and was therefore only assessed in $\mathrm{BH} 2$. MR images were assessed by a single radiologist (A.D.K.) with $>20$ years of experience with the MR imaging of NPC. 


\begin{tabular}{|c|c|}
\hline MR Imaging Features of NPC & Method of Analysis \\
\hline Site & $\begin{array}{l}\text { Walls: mucosa plus submucosa, including the lymphoid tissue layers in the mucosal space superficial } \\
\text { to the fascia and pharyngeal muscles; wall subsites comprise the following: the pharyngeal recess, } \\
\text { roof (above the level of the pharyngeal recess), and posterior/inferior walls (at or below the level } \\
\text { of the pharyngeal recess) } \\
\text { Adenoid: central mucosal lymphoid tissues in the roof and upper posterior wall where lymphoid } \\
\text { tissue within the nasopharynx is abundant } \\
\text { Center: lateral, central, or diffuse }\end{array}$ \\
\hline Size & Volume (area of nasopharyngeal walls and adenoid on each slice $\times$ slice thickness) \\
\hline Size asymmetry & $\begin{array}{l}\text { Area difference between the right and left halves of the nasopharynx on the slice with the } \\
\text { maximum difference, expressed as the } \% \Delta \mathrm{A} \text { (difference in area between the } 2 \text { sides/area on } \\
\text { smaller side } \times 100 \text { ) }\end{array}$ \\
\hline Signal intensity & $\begin{array}{l}\text { Representative ROI is selected on the T1-weighted postcontrast image (T2-weighted signal intensity } \\
\text { is measured at the corresponding site); signal intensity is expressed as a ratio of signal intensity/ } \\
\text { signal intensity of the belly of the lateral pterygoid muscle }\end{array}$ \\
\hline Signal intensity asymmetry & $\begin{array}{l}\text { Subjective signal intensity asymmetry between the right and left halves of the nasopharynx on } \\
\text { Tl-weighted postcontrast images }\end{array}$ \\
\hline Homogeneity & $\begin{array}{l}\text { Subjective assessment of signal intensity on Tl-weighted postcontrast images, divided into } \\
\text { homogeneous and heterogeneous }\end{array}$ \\
\hline Deep mucosal white line & $\begin{array}{l}\text { Line/band of greater contrast enhancement in the deep layer relative to the superficial layer of the } \\
\text { nasopharyngeal wall at the site of the tumor, divided into absent, present with focal loss, and } \\
\text { present and intact; assessment was not made at the adenoid }\end{array}$ \\
\hline Adenoidal septa & $\begin{array}{l}\text { Contrast-enhancing septa within the adenoid, divided into absent, present and distorted } \\
\text { (displacement or partial loss excluding that caused by adenoidal cysts), and present and not } \\
\text { distorted (intact and symmetric) }\end{array}$ \\
\hline
\end{tabular}

\section{Statistical Analysis}

Differences between NPC and BH were compared using logistic regression analyses (odds ratios and corresponding 95\% confidence intervals) of volume, the maximum percentage difference in area $(\% \Delta \mathrm{A})$, and signal intensity, and the $\chi 2$ test to evaluate signal intensity asymmetry, the deep mucosal white line, and adenoidal septa. Differences in signal intensity among NPC, BH1, and $\mathrm{BH} 2$, including a subgroup analysis of signal intensities obtained on the same MR imaging scanner, were compared using the analysis of variance and are depicted with boxplots. A receiver operating characteristic curve analysis and subsequent area under the curve calculation were used to identify the optimal thresholds for volume and $\% \Delta \mathrm{A}$ by maximizing the sensitivity and specificity, and the diagnostic performances of these thresholds were calculated. Five-fold cross-validation was performed to examine the accuracy of the volume and size asymmetry. All statistical tests were 2 -sided, and a $P$ value of $<$ .05 indicated a statistically significant difference. Analyses were performed with SPSS statistical analysis software (Version 23.0; IBM, Armonk, New York).

\section{RESULTS \\ MR Imaging Features of Early-Stage T1 NPC Confined to the Nasopharynx}

NPC involved the adenoid in 111 patients and/or the walls in 183 patients. Affected subsites, including those deep to the adenoid, were the lateral nasopharyngeal recess, the roof, and the posterior/ inferior subsites in 157/189 (83.1\%), 184/189 (97.4\%), and 148/ 189 patients $(78.3 \%)$, respectively. Twenty of 189 patients $(10.6 \%)$ had 1 subsite only, and all subsites were affected bilaterally in $12 / 189$ patients $(6.3 \%)$. The tumor center was lateral in $134 / 189$ patients $(70.9 \%$ ) (Fig 1), of which 95 crossed the midline; central in $25 / 189$ patients (13.2\%) (Fig 2); and diffuse in $30 / 189$ patients $(15.9 \%$ ) (Fig 3). NPCs were enhanced het- erogeneously in 129/189 patients (68.3\%) (Fig 1C). Despite large variations in size, asymmetry, and signal intensity between the 2 halves of the nasopharynx, almost all cases exhibited the deep mucosal white line $(180 / 183,98.4 \%)$ (Figs $1 A,-B$ and $3 B$ ), which was interrupted in at least 1 slice in $153 / 180$ patients $(85 \%)$ (Fig $1 B)$. Furthermore, the adenoidal septa were absent/distorted in $111 / 111$ patients (100\%) with an adenoid (Fig 2). Further details are presented in the On-line Table.

\section{MR Imaging Features of $\mathrm{BH}$}

BH1 involved the walls in all 66/66 patients (100\%) (Fig 4A), and no adenoid without contrast-enhancing septa was observed. BH2 involved the walls, adenoid, and both sites in 17/78 (22\%), 5/78 (6\%), and 56/78 patients (72\%), respectively (Fig 4B, -C). Size (volume and size asymmetry), signal intensity (intensity and signal asymmetry), deep mucosal white line, and adenoidal septa data are listed in the On-line Table.

\section{Comparison of the MR Imaging Features of Early-Stage T1 NPC and BH}

The NPC signal intensity fell between those of $\mathrm{BH} 1$ and $\mathrm{BH} 2$ (T1 postcontrast $\mathrm{BH} 1>\mathrm{NPC}>\mathrm{BH} 2$; $\mathrm{T} 2 \mathrm{BH} 2>\mathrm{NPC}>\mathrm{BH} 1)$, and significant differences were observed among the 3 groups $(P<$ .001) (On-line Table and Fig 5). A subgroup analysis of cases performed on the same 1.5T MR imaging scanner $(\mathrm{NPC}=31$, $\mathrm{BH} 1=43, \mathrm{BH} 2=64)$ revealed the same signal intensity differences among the 3 groups, which remained significant for all T1 postcontrast scans (BH1 versus $\mathrm{BH} 2, P<.001 ; \mathrm{BH} 1$ versus NPC, $P<.001$; BH2 versus NPC, $P=.015)$ and T2 comparisons of $\mathrm{BH} 1$ versus $\mathrm{BH} 2(P<.001)$ and $\mathrm{BH} 2$ versus NPC $(P=.018)$, but not $\mathrm{BH} 1$ versus $\mathrm{NPC}(P=.183)$.

Compared with $\mathrm{BH}$, stage T1 NPC confined to the nasopharynx exhibited a significantly greater volume, size asymmetry, sig- 

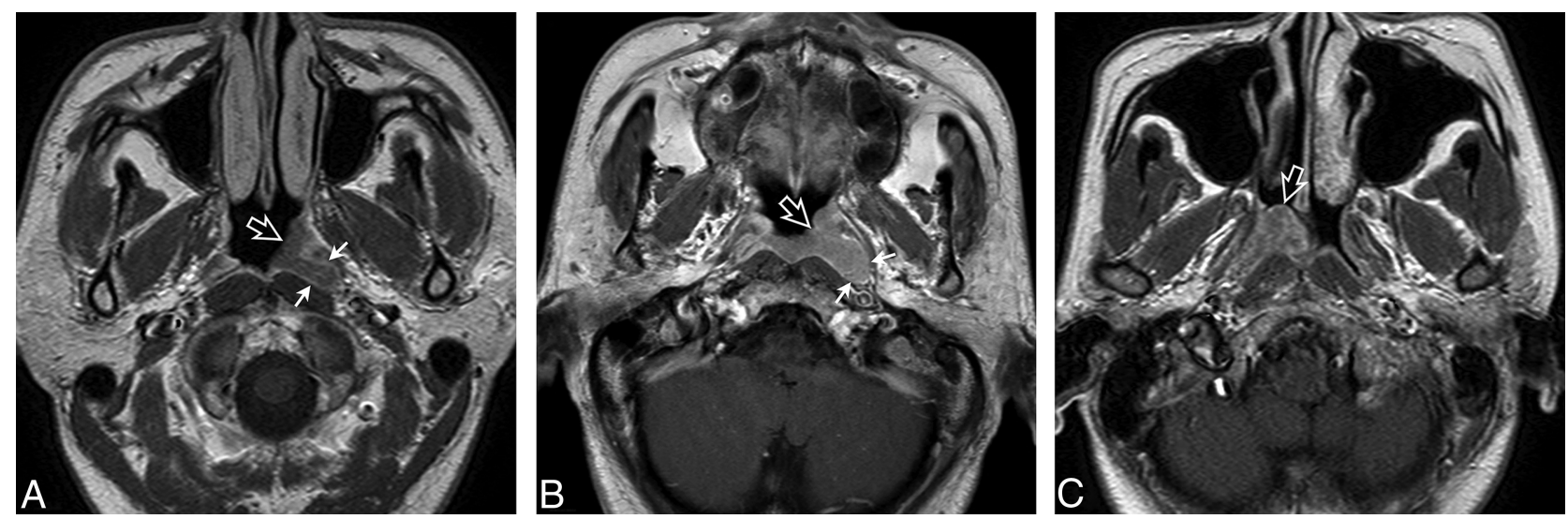

FIG 1. A, Axial T1-weighted postcontrast MR image of the nasopharynx in a 51-year-old man with NPC (open arrow). An asymmetric tumor with a lateral center at the level of the left pharyngeal recess is confined to 1 side of the nasopharynx. The tumor exhibits homogeneous low contrast enhancement with an intact deep mucosal white line (small solid arrows) along the deep margin. $B$, Axial T1-weighted postcontrast MR image of the nasopharynx in a 68-year-old man with NPC (open arrow). An asymmetric tumor with a lateral center at the level of the left pharyngeal recess is confined to 1 side of the nasopharynx. The tumor exhibits homogeneous moderate contrast enhancement with a focal loss of the deep mucosal white line (small solid arrows) along the deep margin. C, Axial T7-weighted postcontrast MR image of the nasopharynx in a 44-year-old woman with NPC (open arrow). An asymmetric tumor with a lateral center at the level of the right side of the roof shows heterogeneous contrast enhancement.

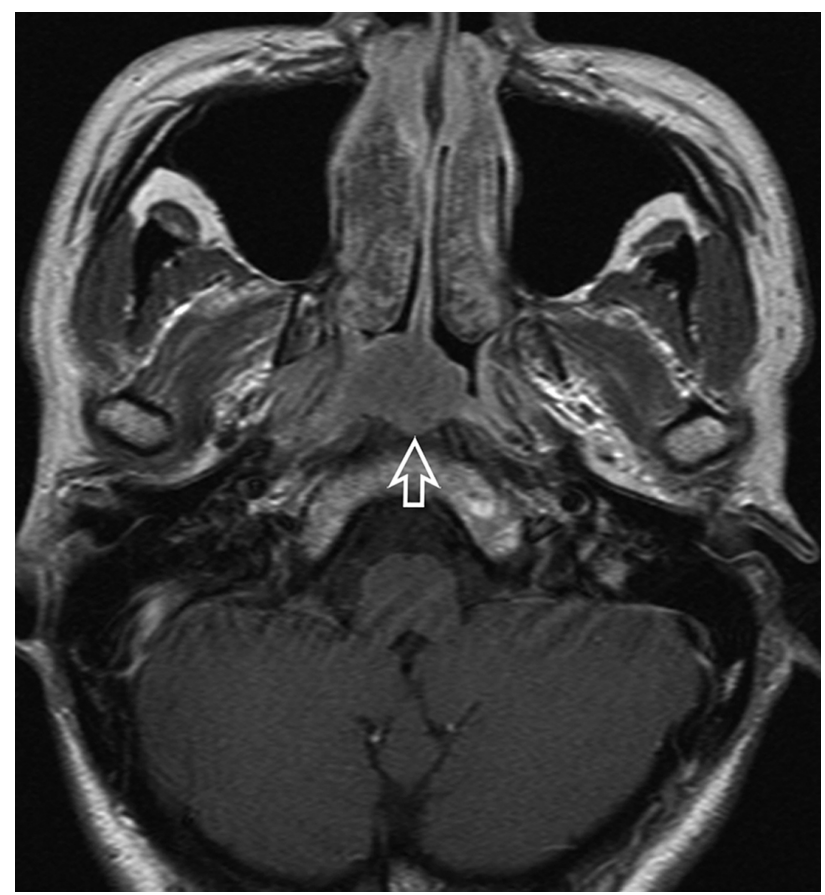

FIG 2. Axial TI-weighted postcontrast MR image of the nasopharynx in a 32-year-old woman with NPC (open arrow). An asymmetric tumor with a central center in the adenoid exhibits homogeneous contrast enhancement and loss of the normal adenoidal septa.

nal asymmetry, focal loss of the deep mucosal white line, and absence/distortion of the adenoidal septa $(P<.001)$ (On-line Table). The optimum NPC detection thresholds were a volume of $\geq 5.01 \mathrm{~cm}^{3}(P<.001$, area under the receiver operating characteristic curve $=0.676)$ and a $\% \Delta \mathrm{A}$ of $\geq 33.7 \%(P<.001$, area under the receiver operating characteristic curve $=0.948)($ Fig 6). The diagnostic performance of MR imaging stage T1 NPC detection based on volume, $\% \Delta \mathrm{A}$, signal asymmetry, deep mucosal white line absence/focal loss, and adenoidal septa absence/distortion is shown in Table 2. The estimated accuracy of volume and size asymmetry obtained from 5-fold cross-validation was not overestimated and was within the $95 \%$ CI of observed accuracy of volume and size asymmetry. Although a $\% \Delta \mathrm{A}$ of $\geq 33.7 \%$ was the most accurate criterion for NPC detection, this parameter yielded $22(11.6 \%)$ false-negatives among 189 cases and 13 (9.0\%) falsepositives among 144 cases. False-negative results arose in NPCs that were diffuse $(n=12)$ (Fig 3$)$, central $(n=8)$, or lateral $(n=$ 2 ) and had a $\% \Delta \mathrm{A}$ of $2.4 \%-31.0 \%$ (mean, $15.5 \%$ ). False-positive results arose in asymmetric $\mathrm{BHs}$ with $\% \Delta \mathrm{A}$ values of $34.6 \%$ $82.3 \%$ (mean, 51.9\%) (Fig 7). A subgroup analysis of the diagnostic performances of criteria in symmetric $(\% \Delta \mathrm{A}<33.7 \%) \mathrm{NPC}$ and $\mathrm{BH}$ showed the highest level of accuracy for a focal loss of the mucosal white line $(90.7 \%)$, followed by absent/distorted adenoidal septa $(89.3 \%)$, signal asymmetry $(83.0 \%)$, and volume (71.2\%) (Table 3).

\section{DISCUSSION}

Plasma markers are currently used to screen patients at risk of NPC, including asymptomatic subjects from regional populations and family screening programs as well as symptomatic patients with nonspecific symptoms such as epistaxis. In this setting, MR imaging is also used to detect clinically occult early stage NPC in patients with abnormal blood test results but normal endoscopic results. Unfortunately, it is common to encounter patients who have both false-positive NPC plasma markers and $\mathrm{BH}$ of the nasopharyngeal walls and adenoid. In these patients, the challenge is not only to detect stage T1 NPC confined to the nasopharynx using MR imaging but also to discriminate these cancers from benign disease and thus reduce the number of unnecessary nasopharyngeal biopsies.

Although detailed MR imaging patterns of the local spread of NPCs have been well-described in the literature, ${ }^{9-11}$ there is a paucity of data related to stage T1 NPC confined to the nasopharynx, despite its importance for NPC screening. In this study, we found that stage T1 NPCs varied considerably in size, from small tumors localized at a single site to large exophytic tumors involv- 
ing all sites and filling the nasopharyngeal cavity. Most NPCs arose laterally to cause size and signal asymmetries between the nasopharyngeal halves, whereas less than a third were diffuse or located centrally in the roof or posterior wall. The deep mucosal white line along the deep tumor margin was almost universally present among stage T1 cancers. This deep mucosal white line of greater contrast enhancement in the deep relative to the superficial layer has received scant attention in the literature. We believe that this line represents contrast enhancement in the dense fibrous tissue containing submucosal blood vessels. In most NPCs (84\%), the deep mucosal white line exhibited focal discontinuity on at least 1 imaging slice. Although the integrity of this line has not been previously assessed in NPC, we believe that discontinuity might represent the earliest sign of impending extranasopharyn-
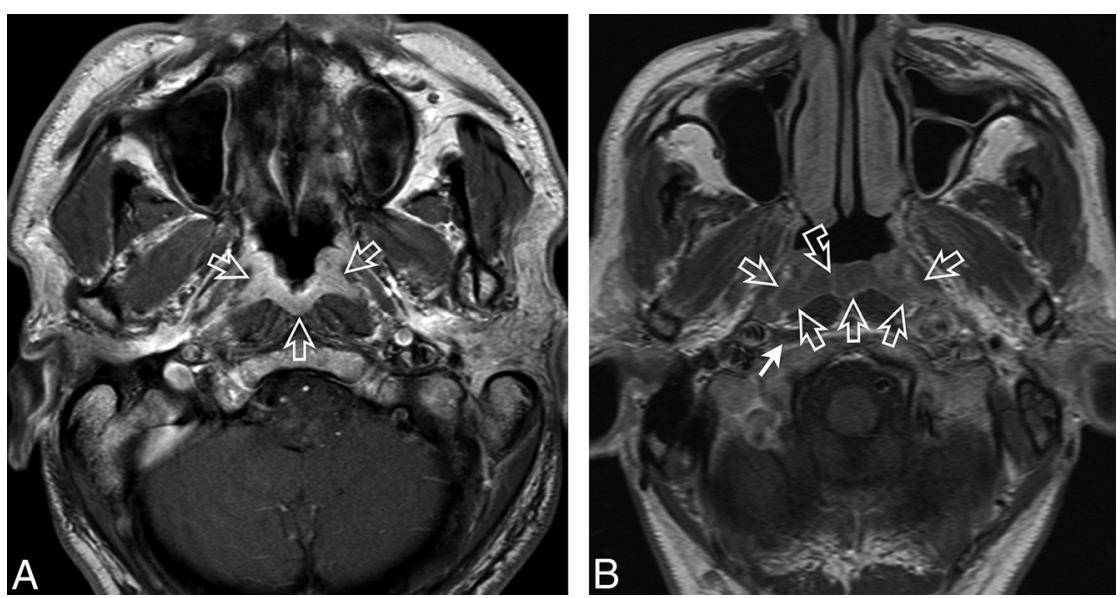

FIG 3. A, Axial T1-weighted postcontrast MR image of the nasopharynx in a 51-year-old man with NPC (open arrows). A symmetric tumor with diffuse involvement of all nasopharyngeal walls exhibits homogeneous contrast enhancement without a white line along the deep mucosal margin. B, Axial Tl-weighted postcontrast MR image of the nasopharynx in a 59-year-old man with NPC (open arrows). A symmetric tumor with diffuse involvement of the nasopharyngeal walls exhibits homogeneous low contrast enhancement and an intact mucosal white line along the deep margin. The adenoid extends along the posterior wall from the roof, with an adenoidal "stripe" on the right (curved open arrow) but not on the left side. A small right retropharyngeal node is also indicated (solid arrow). The patient had bulky N3-stage metastatic nodes below this level.
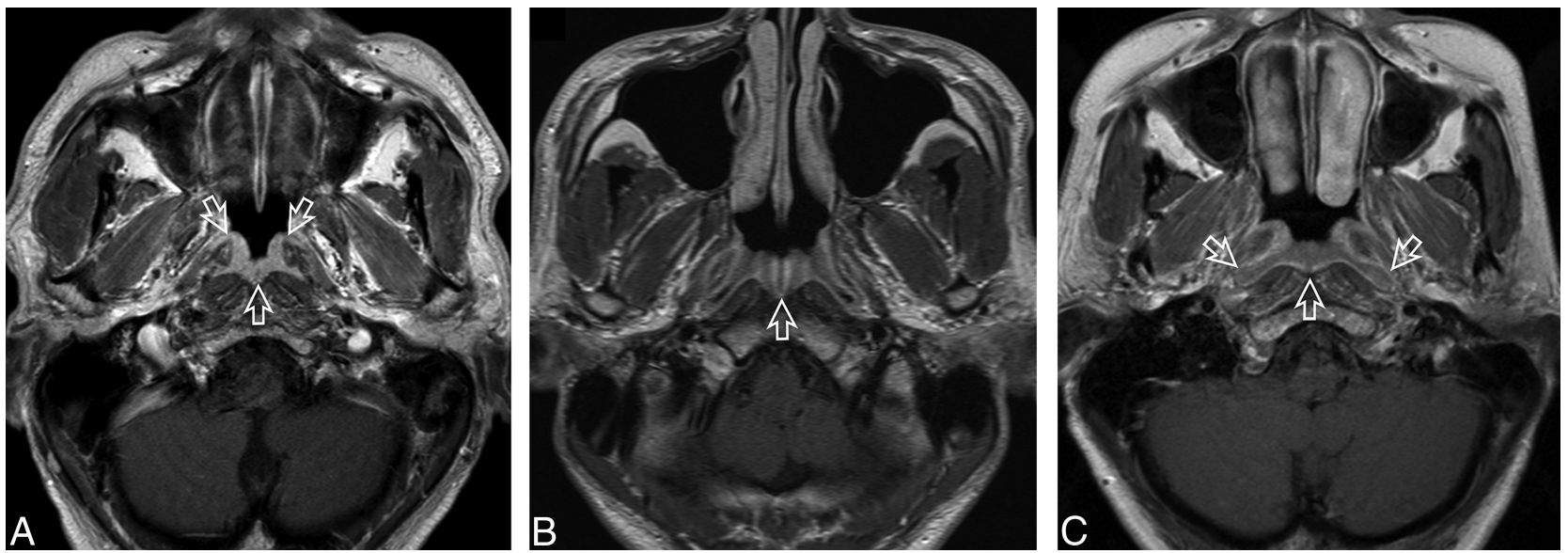

FIG 4. A, Axial T1-weighted postcontrast MR image of the nasopharynx in a 53-year-old man with BH1 (open arrows). An area of diffuse symmetric mucosal thickening with homogeneous contrast enhancement is visible. $B$, Axial T1-weighted postcontrast MR image of the nasopharynx in a 28-year-old man with BH2 of the adenoid (open arrow). The symmetric lesion exhibits contrast-enhancing septa that run perpendicular to the nasopharyngeal wall and are separated by columns of low contrast enhancement. $C$, Axial TI-weighted postcontrast MR image of the nasopharynx in a 48-year-old woman with $\mathrm{BH} 2$ along the nasopharyngeal walls (open arrows). An area of diffuse, symmetric homogeneous low contrast enhancement and an intact deep mucosal white line along the deep margin are visible. 

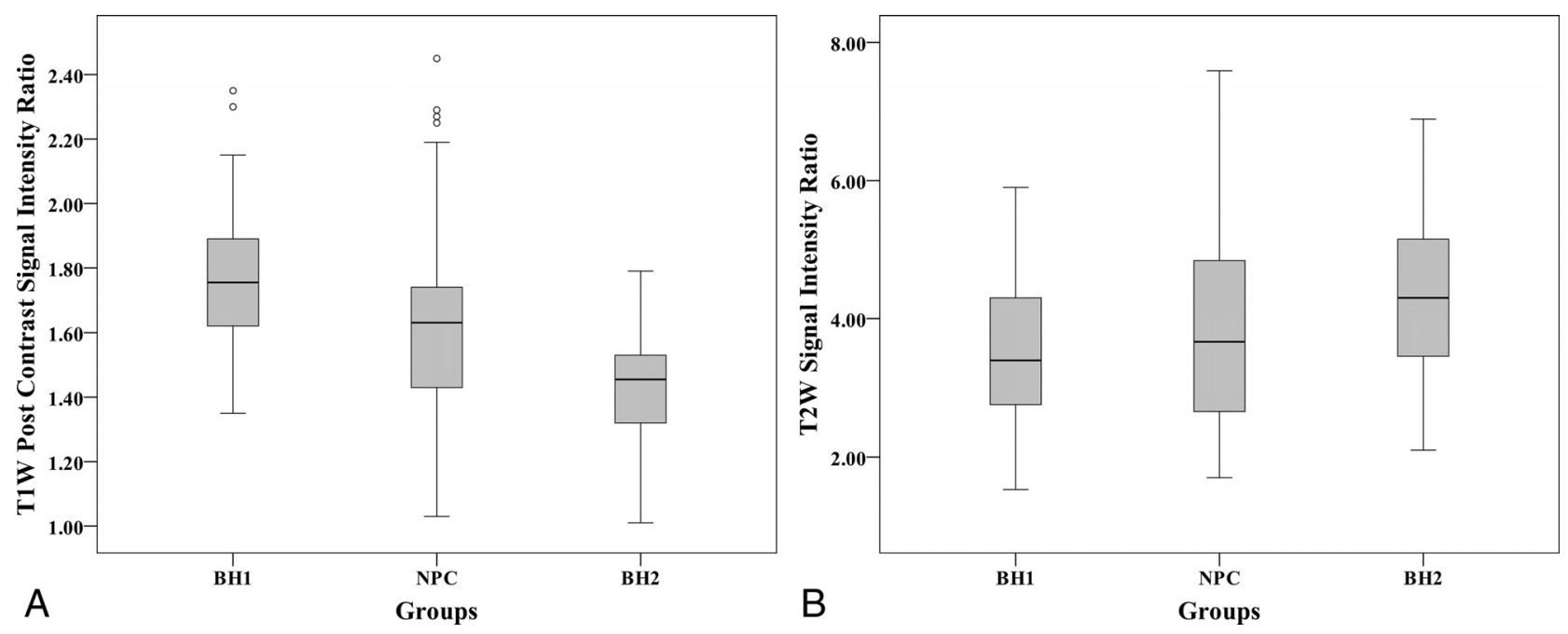

FIG 5. A, Boxplots showing differences in the $T 1$ postcontrast signal intensity ratios (relative to muscle) among NPC, BH1, and BH2. B, Boxplots show differences in the $\mathrm{T} 2$ signal intensity ratios (relative to muscle) among $\mathrm{NPC}, \mathrm{BH} 1$, and $\mathrm{BH} 2$.

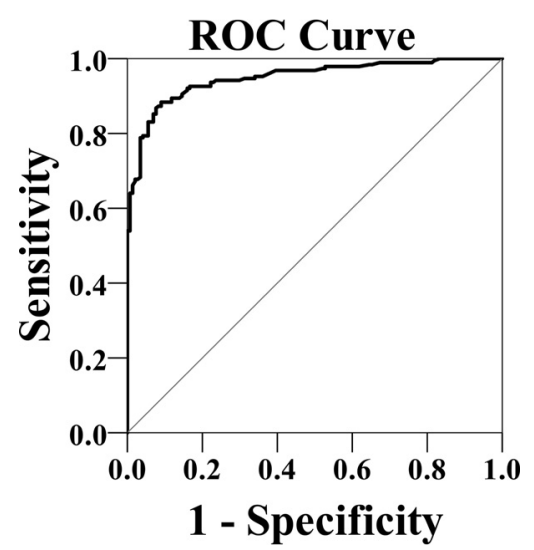

FIG 6. Receiver operating characteristic curve of the percentage difference in area for NPC detection.

T1 NPCs exhibited variable contrast enhancement both within heterogeneous tumors and among different tumors. $\mathrm{BH}$ is also known to exhibit a range of MR imaging appearances. ${ }^{2,13-17}$ Notably, there were no differences between NPC and BH in this study when the signal intensities of $\mathrm{BH} 1$ and $\mathrm{BH} 2$ were combined. The range of NPC contrast-enhancement levels and the overlap with $\mathrm{BH}$ are of concern regarding symmetric NPCs, which can exhibit higher contrast enhancement and thus appear like BH1 (Figs $3 A$ and $4 A$ ) or lower contrast enhancement and thus appear like BH2 (Figs $3 B$ and $4 C$ ). Signal intensity was also assessed on the basis of the asymmetry in signal intensity between the 2 sides of the nasopharynx. Although this criterion was highly accurate for NPC, the positive predictive value was low, especially in symmetric NPCs (positive predictive value, 42\%). Volume was the poorest discriminator among all criteria, even for symmetric NPCs, because the volumes of both NPC and BH ranged widely. Therefore, the use of volume to determine NPC would have led to a high number of nasopharyngeal biopsies for benign disease.

The 2 remaining MR imaging criteria related to the adenoidal septa (adenoid) and the deep mucosal white line (walls) exhibited greater promise for NPC detection. These 2 criteria detected NPC with a high level of accuracy, including symmetric tumors that may otherwise have been missed by MR imaging. Regarding the first criterion, distortion, disruption, or a complete loss of the adenoidal septa was significantly more likely to appear in NPC compared with adenoidal BH. However, the adenoidal septa should be assessed carefully because distortion caused by cysts or a single central column of lymphoid hyperplasia could be mistaken for NPC. ${ }^{15}$ Accordingly, vestigial adenoids and those comprising mainly cysts were not assessed in this study. Moreover, some previous MR imaging studies reported a higher rate of absent adenoidal septa in cases of adenoidal BH. ${ }^{17}$ Therefore, we suggest that an hypertrophied adenoid with absent or distorted septa should only be biopsied in a patient who is a a high risk of NPC and has undergone an MR imaging examination dedicated to the nasopharynx. However, the previously reported high negative predictive value of this sign suggests that nasopharyngeal biopsy can be avoided if the adenoidal septa are symmetric and separated by hyperplastic columns of low contrast enhancement (ie, striped appearance). ${ }^{2}$ Regarding the second criterion, a focal loss of the deep mucosal white line was found to be significantly more common in NPC than in BH2. However, any assessment of the loss of this deep mucosal white line should be mindful of the fact that this line is not seen in the BH1 pattern. The $\mathrm{BH} 1$ and $\mathrm{BH} 2$ patterns often coexist in the nasopharynx; therefore, a focal loss of this line should only be determined on a slice through the nasopharynx where the line is present but focally disrupted.

Despite the use of new criteria, the ability of MR imaging to discriminate NPC from BH remains hindered by the false-positive findings of NPC, which are caused by asymmetric $\mathrm{BH}$. The presence of a focal area of $\mathrm{BH} 2$ (Fig 7) is of special concern because the deep mucosal white line and superficial band of low contrast enhancement show a striking similarity to some smallstage T1 NPCs (Fig 1A). Accordingly, we have continued to follow these subjects. We believe that the diffuse $\mathrm{BH} 2$ pattern might be a response to stimulants such as Epstein-Barr virus infection, which cause a greater abundance of lymphoid cells in the lymphoid stroma and epithelium and may cause the appearance of a band of 
Table 2: Diagnostic performance of MR imaging for the discrimination of early NPC from BH

\begin{tabular}{|c|c|c|c|c|c|}
\hline & Volume & Size Asymmetry & Signal Asymmetry & $\begin{array}{l}\text { Deep Mucosal } \\
\text { White Line }\end{array}$ & Adenoid \\
\hline \multirow[t]{2}{*}{ Test positive for NPC } & Volume $\geq 5.01 \mathrm{~cm}^{3}$ & $\% \Delta \mathrm{A} \geq 33.7 \%$ & $\begin{array}{l}\text { Signal asymmetry } \\
\text { present }\end{array}$ & $\begin{array}{l}\text { Focal loss of the } \\
\text { deep mucosal } \\
\text { white line }\end{array}$ & $\begin{array}{l}\text { Septa absent or } \\
\text { distorted } \\
\text { (excluding septa } \\
\text { distorted by cysts) }\end{array}$ \\
\hline & $\begin{array}{c}n=333(\mathrm{NPC}=189, \\
\mathrm{BH}=144)\end{array}$ & $\begin{array}{c}n=333(\mathrm{NPC}=189 \\
\mathrm{BH}=144)\end{array}$ & $\begin{array}{c}n=333(\mathrm{NPC}=189 \\
\mathrm{BH}=144)\end{array}$ & $\begin{array}{c}n=253(\mathrm{NPC}=18 \\
\mathrm{BH} 2=73)\end{array}$ & $\begin{array}{c}n=172(\mathrm{NPC}=111 \\
\mathrm{BH} 2=61)\end{array}$ \\
\hline Area under the curve $(95 \% \mathrm{Cl})$ & $0.676(0.618-0.734)$ & $0.948(0.926-0.971)$ & & & \\
\hline$P$ value & $<.001$ & $<.001$ & & & \\
\hline True-positive (No.) & 119 & 167 & 161 & 153 & 111 \\
\hline False-positive (No.) & 47 & 13 & 26 & 10 & 9 \\
\hline True-negative (No.) & 97 & 131 & 118 & 63 & 52 \\
\hline False-negative (No.) & 70 & 22 & 28 & 27 & 0 \\
\hline Sensitivity (\%) & 63.0 & 88.4 & 85.2 & 85.0 & 100.0 \\
\hline Specificity (\%) & 67.4 & 91.0 & 81.9 & 86.3 & 85.2 \\
\hline Positive predictive value (\%) & 71.7 & 92.8 & 86.1 & 93.9 & 92.5 \\
\hline Negative predictive value (\%) & 58.1 & 85.6 & 80.8 & 70.0 & 100.0 \\
\hline Accuracy $(\%)$ & 64.9 & 89.5 & 83.8 & 85.4 & 94.8 \\
\hline
\end{tabular}

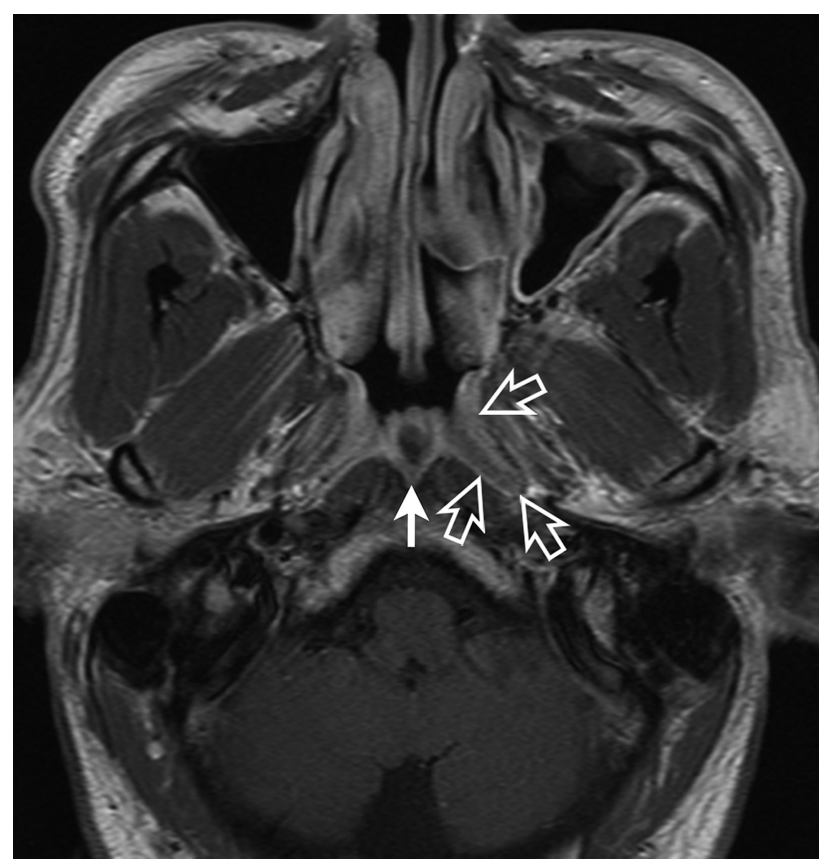

FIG 7. Axial T1-weighted postcontrast MR image of the nasopharynx in a 52-year-old man with asymmetric BH. Greater thickening is observed in the left side of the roof (open arrows), where a focal area of mucosal thickening comprises a superficial band of low contrast enhancement overlying an intact deep mucosal white line (BH type 2). A Tornwaldt cyst is also present (solid arrow).

low contrast enhancement in the superficial layer of the nasopharyngeal mucosa, as well as in the columns of the hypertrophied adenoid. Differential rates of resolution might explain the asymmetry and why a minority of $\mathrm{BH} 2$ cases are focal by the time the MR imaging examination is performed. These focal sites of $\mathrm{BH} 2$ might act as a chronic reservoir of infection and become the MR imaging precursors of NPC. Furthermore, recruitment of vessels by the growing tumor might result in areas of greater enhancement like those observed in BH1and explain the heterogeneous enhancement pattern of early T-stage NPC. Although currently this scenario is only conjecture, the $\mathrm{BH} 2$ pattern is interesting and we believe it deserves future study.
Size asymmetry was the best overall criterion for detecting stage T1 NPC; however, size asymmetry included not only subjects with a focal mass but also those with asymmetry in an otherwise diffuse lymphoid hyperplasia pattern. Our previous NPC detection study with long-term follow-up ${ }^{3}$ showed that asymmetry in a diffuse benign hyperplasia pattern without a focal mass had a low specificity for NPC; therefore, to keep nasopharyngeal biopsies of healthy subjects to a minimum, we did not use this criterion to indicate NPC in a recent population screening study. ${ }^{7}$ Taking into consideration the new criteria in this study and recent research, we have updated our proposed MR imaging grading system (Table 4): The grades for normal (grade 1) and probably benign (grade 2) are unchanged; asymmetry in thickness of an otherwise benign hyperplasia pattern is now graded as indeterminate (grade 3); the new signs (asymmetry in signal, focal loss of the white line, or absence/distortion of adenoidal septa) together with a focal mass are graded as suspicious (grade 4); and extension outside the nasopharynx is graded as probably malignant (grade 5). Future studies are required to validate this proposed MR imaging grading system, but we hope it will provide a framework for further refinement and inclusion of new indicators of malignancy such as the presence of metastatic retropharyngeal nodes.

This study had several limitations. First, the signal intensity in an NPC may be influenced by the subjective choice of the representative ROI and possibly by postbiopsy effects that are not obvious on the images. Differences in magnet strengths and between manufacturers must also be considered as potential limitations when comparing signal ratios. Given these potential limitations, we only used the signal intensity data to illustrate the main differences among the 3 groups (NPC, BH1, and $\mathrm{BH} 2$ ) and not to identify diagnostic thresholds. In addition, a subgroup analysis of cases performed on the same scanner yielded results similar to those of the overall analysis, except for the difference in T2 signal intensity ratios between NPC and BH1, which showed the same trend but no longer reached statistical significance. Second, our results may not be applicable to MR imaging examinations that are not dedicated to the nasopharynx, such as examinations of the brain, which 
Table 3: MR imaging criteria subgroup analysis of symmetric $(\% \Delta A<33.7 \%)$ NPC and BH

\begin{tabular}{|c|c|c|c|c|}
\hline & Volume & Signal Asymmetry & Deep Mucosal White Line & Adenoid \\
\hline \multirow[t]{2}{*}{ Test positive for NPC } & Volume $\geq 5.01 \mathrm{~cm}^{3}$ & Signal asymmetry present & $\begin{array}{l}\text { Focal loss of the deep } \\
\text { mucosal white line }\end{array}$ & $\begin{array}{l}\text { Septa absent or distorted } \\
\text { (excluding septa distorted } \\
\text { by cysts) }\end{array}$ \\
\hline & $\begin{array}{l}n=153(\mathrm{NPC}=22, \\
\mathrm{BH}=131)\end{array}$ & $\begin{array}{l}n=153(\mathrm{NPC}=22 \\
\mathrm{BH}=131)\end{array}$ & $\begin{array}{l}n=83(\mathrm{NPC}=20 \\
\mathrm{BH} 2=63)\end{array}$ & $\begin{array}{l}n=72(\mathrm{NPC}=16 \\
\mathrm{BH} 2=56)\end{array}$ \\
\hline True-positive (No.) & 20 & 11 & 16 & 16 \\
\hline False-positive (No.) & 42 & 15 & 8 & 8 \\
\hline True-negative (No.) & 89 & 116 & 55 & 48 \\
\hline False-negative (No.) & 2 & 11 & 4 & 0 \\
\hline Sensitivity (\%) & 90.1 & 50.0 & 80.0 & 100.0 \\
\hline Specificity (\%) & 67.9 & 88.5 & 87.3 & 85.7 \\
\hline Positive predictive value (\%) & 32.3 & 42.3 & 66.7 & 66.7 \\
\hline Negative predictive value (\%) & 97.8 & 91.3 & 93.2 & 100 \\
\hline Accuracy $(\%)$ & 71.2 & 83.0 & 85.5 & 88.9 \\
\hline
\end{tabular}

Table 4: Proposed MR imaging criteria for primary NPC detection

\begin{tabular}{|c|c|c|}
\hline Grade & Condition & Appearance \\
\hline 1 & Normal & Thin mucosa $\leq 3 \mathrm{~mm}$; adenoid absent, vestigial or composed almost entirely of cysts \\
\hline 2 & Probably benign & $\begin{array}{l}\text { Diffusely thickened }>3 \text {-mm symmetric mucosa either homogeneously enhancing (benign hyperplasia } \\
\text { type 1) or with greater contrast enhancement in the deep layer (mucosal white line) compared with the } \\
\text { superficial layer (benign hyperplasia type 2); adenoid with intact contrast-enhancing septa (benign } \\
\text { hyperplasia type 2) }\end{array}$ \\
\hline 3 & Indeterminate & Diffusely thickened $>3-\mathrm{mm}$ mucosa asymmetric in thickness; adenoid asymmetric in thickness \\
\hline 4 & Suspicious & $\begin{array}{l}\text { 1) Diffusely thickened }>3-\mathrm{mm} \text { mucosa asymmetric in signal intensity; adenoid asymmetric in } \\
\text { signal intensity } \\
\text { 2) Diffusely thickened mucosa }>3 \mathrm{~mm} \text { with focal loss of the mucosal white line; } \\
\text { absence/distortion of the thin contrast-enhancing adenoidal septa (not attributed to cysts) } \\
\text { 3) Focal mass confined within the nasopharynx }\end{array}$ \\
\hline 5 & Probably malignant & $\begin{array}{l}\text { Superficial extension to the nasal cavity/oropharynx or deep extension to the sites bordering } \\
\text { the nasopharynx }\end{array}$ \\
\hline
\end{tabular}

often include sections through the nasopharyngeal roof. Finally, we did not examine the role of diffusion-weighted imaging, which remains in the early stages of development for small nasopharyngeal lesions, or the combined performance of MR imaging and nasopharyngeal endoscopy and plasma levels of Epstein-Barr virus DNA.

\section{CONCLUSIONS}

In this era of plasma marker screening for NPC, MR imaging is assuming a greater role in the detection of endoscopically occult stage T1 disease confined to the nasopharyngeal mucosa or adenoid. We found that although stage T1 NPCs varied in size, most were centered laterally, thus causing asymmetries in size and signal intensity, and nearly all exhibited a mucosal white line deep in the tumor that was frequently interrupted and absent or distorted adenoidal septa. Compared with $\mathrm{BH}$, stage T1 NPC exhibited significantly greater volume, size, and signal intensity asymmetries; focal losses of the deep mucosal white line; and absence/distortion of the adenoidal septa. The contrast enhancement of stage T1 NPC was weaker than that of $\mathrm{BH} 1$ and stronger than that of $\mathrm{BH} 2$, though considerable overlap was observed. Although size asymmetry remained the best criterion for identifying stage T1 NPC, the ability of MR imaging to detect stage T1 NPC was reduced for symmetric tumors that were diffuse or centrally located and shared overlapping signal intensities with those of $\mathrm{BH}$. For these cancers, the focal loss of the deep mucosal white line and absent/distorted adenoidal stripes were the best criteria for NPC detection, fol- lowed by signal asymmetry. Therefore, a range of MR imaging criteria is needed to detect stage T1 NPC, and we have included these in our proposed guidelines. Future studies are needed to validate and refine these criteria.

\section{ACKNOWLEDGMENTS}

We would like to acknowledge the assistance of Pak Lai Terry Tsoi, Way Loon Jonathan Chen, Helen Pui Yan Lee, and Man Tung Karen Yuen.

Disclosures: Ann D. King—RELATED: Grant: Research Grants Council of the Hong Kong Special Administrative Region, China, Comments: partially funded by grants (project No. CUHK4457/06, CUHK4656/12, and SEG_CUHK02)*. Benjamin King Hong Law_RELATED: Grant: Research Grants Council of the Hong Kong Special Administrative Region, China, Comments: partially funded by grants (project No. CUHK4457/06, CUHK4656/12, and SEG_CUHK02).* Khoon Leong ChuahUNRELATED: Employment: Tan Tock Seng Hospital, Comments: employed as a consultant pathologist. Frankie K.F. Mo-RELATED: Grant: Research Grants Council of the Hong Kong Special Administrative Region, China, Comments: partially funded by grants (project No. CUHK4457/06, CUHK4656/12, and SEG_CUHK02)*. K.C. Allen Chan—RELATED: Grant: Kadoorie Charitable Foundation, Comments: I received funding support from Kadoorie Charitable Foundation for NPC research*; UNRELATED: Board Membership: Cirina, Comments: I am a director of Cirina; Payment for Lectures Including Service on Speakers Bureaus: Bio-Rad, Comments: I received an honorarium from Bio-Rad as an invited speaker in academic lectures; Patents (Planned, Pending or Issued): Cirina, Comments: I hold patents on molecular diagnostics; Royalties: Cirina; Stock/Stock Options: Cirina, Comments: I hold equities in Cirina. Anthony T.C. Chan—RELATED: Grant: Research Grants Council of the Hong Kong Special Administrative Region, China, Comments: partially funded by grants (project No. CUHK4457/06, CUHK4656/12, and SEG_CUHK02)*. Alexander C. Vlantis - RELATED: Grant: Research Grants Council of the Hong Kong Special Administrative Region, China, Comments: partially funded by grants (project 
No. CUHK4457/06, CUHK4656/12, and SEG_CUHK02)*. *Money paid to the institution.

\section{REFERENCES}

1. King AD, Vlantis AC, Tsang RK, et al. Magnetic resonance imaging for the detection of nasopharyngeal carcinoma. AJNR Am J Neuroradiol 2006;27:1288-91 Medline

2. King $\mathrm{AD}$, Vlantis $\mathrm{AC}$, Bhatia $\mathrm{KS}$, et al. Primary nasopharyngeal carcinoma: diagnostic accuracy of MR imaging versus that of endoscopy and endoscopic biopsy. Radiology 2011;258:531-37 CrossRef Medline

3. King AD, Vlantis AC, Yuen TW, et al. Detection of nasopharyngeal carcinoma by MR imaging: diagnostic accuracy of MRI compared with endoscopy and endoscopic biopsy based on long-term followup. AJNR Am J Neuroradiol 2015;36:2380 - 85 CrossRef Medline

4. Lo YM, Chan AT, Chan LY, et al. Molecular prognostication of nasopharyngeal carcinoma by quantitative analysis of circulating Epstein-Barr virus DNA. Cancer Res 2000;60:6878-81 Medline

5. Leung SF, Tam JS, Chan AT, et al. Improved accuracy of detection of nasopharyngeal carcinoma by combined application of circulating Epstein-Barr virus DNA and anti-Epstein-Barr viral capsid antigen IgA antibody. Clin Chem 2004;50:339-45 CrossRef Medline

6. Chan KC, Hung EC, Woo JK, et al. Early detection of nasopharyngeal carcinoma by plasma Epstein-Barr virus DNA analysis in a surveillance program. Cancer 2013;119:1838-44 CrossRef Medline

7. Chan KC, Woo JK, King A, et al. Analysis of plasma Epstein-Barr virus DNA to screen for nasopharyngeal cancer. N Engl J Med 2017; 377:513-22 CrossRef Medline

8. Wu YP, Cai PQ, Tian L, et al. Hypertrophic adenoids in patients with nasopharyngeal carcinoma: appearance at magnetic reso- nance imaging before and after treatment. Chin J Cancer 2015;34: 130-36 CrossRef Medline

9. Ng SH, Chang TC, Ko SF, et al. Nasopharyngeal carcinoma: MRI and CT assessment. Neuroradiology 1997;39:741-46 CrossRef Medline

10. King AD, Lam WW, Leung SF, et al. MRI of local disease in nasopharyngeal carcinoma: tumour extent vs tumour stage. Br J Radiol 1999; 72:734-41 CrossRef Medline

11. Liang SB, Sun Y, Liu LZ, et al. Extension of local disease in nasopharyngeal carcinoma detected by magnetic resonance imaging: improvement of clinical target volume delineation. Int J Radiat Oncol Biol Phys 2009;75:742-50 CrossRef Medline

12. Gao Y, Zhu SY, Dai Y, et al. Diagnostic accuracy of sonography versus magnetic resonance imaging for primary nasopharyngeal carcinoma. J Ultrasound Med 2014;33:827-34 CrossRef Medline

13. Vogler RC, Ii FJ, Pilgram TK. Age-specific size of the normal adenoid pad on magnetic resonance imaging. Clin Otolaryngol Allied Sci 2000;25:392-95 CrossRef Medline

14. Kösling S, Knipping S, Hofmockel T. Imaging of nasopharyngeal diseases [in German]. Radiologe 2009;49:17-26 CrossRef Medline

15. Bhatia KS, King AD, Vlantis AC, et al. Nasopharyngeal mucosa and adenoids: appearance at MR imaging. Radiology 2012;263:437-43 CrossRef Medline

16. Sekiya K, Watanabe M, Nadgir RN, et al. Nasopharyngeal cystic lesions: Tornwaldt and mucous retention cysts of the nasopharynx: findings on MR imaging. J Comput Assist Tomogr 2014;38:9-13 CrossRef Medline

17. Surov A, Ryl I, Bartel-Friedrich S, et al. MRI of nasopharyngeal adenoid hypertrophy. Neuroradiol J 2016;29:408-12 CrossRef Medline 